\title{
Features of Entrainment of Spinal Pattern Generators for Locomotor Activity in the Lamprey Spinal Cord
}

\author{
Andrew D. McClellan and Karen A. Sigvardt \\ Department of Physiology and Biophysics, University of lowa, lowa City, lowa 52242, and Department of Neurology, \\ University of California-Davis, Veterans Administration Medical Center, Martinez, California 94553
}

\begin{abstract}
The in vitro lamprey spinal cord contains a "central pattern generator" (CPG) that can generate locomotor activity with excitatory amino acids added to the bath. The motor pattern can be entrained by imposed rhythmic bending of either the caudal or rostral end of the notochord/spinal cord. In the present study, the quantitative and mechanistic features of entrainment were investigated. Increasing the amplitude of the imposed movement increased the range of frequencies over which entrainment occurred. Brief, pulsed, imposed movements could reset the locomotor rhythm. During entrainment at different imposed movement frequencies, the burst duration was a constant proportion of about $35 \%$ of the cycle time. The intersegmental phase lag, which is usually constant at about $\mathbf{0 . 0 1}$ during locomotion, decreased significantly with caudal imposed movements. A small increase in the phase lags was observed with rostral imposed movements.
\end{abstract}

In low-calcium Ringer's, briefly bending the notochord/ spinal cord activated intraspinal mechanoreceptors and elicited ascending and descending unit activity in lateral spinal fascicles many segments from the point of bending. However, this ascending or descending movement-related activity was insufficient to fully entrain the locomotor rhythm, since blocking the pattern in the region of bending abolished 1:1 entrainment. The mechanoreceptors appear to act locally on the CPG networks, since interrupting the ascending or descending movement-related activity with lesions of the lateral fascicles did not abolish entrainment. In contrast, stripping the very lateral margins of the spinal cord in the region of bending did abolish entrainment, presumably by destroying the transduction region of the mechanoreceptors. The data, taken together, suggest that the mechanoreceptors entrain the local CPG networks, and this timing information is then distributed to the other parts of the spinal motor networks through the coordinating system.

\footnotetext{
Received Jan. 27, 1987; revised June 29, 1987; accepted July 21, 1987.

We would like to thank S. Grillner, P. Stein, and G. Richerson for reviewing an earlier version of the manuscript, and $\mathrm{Mr}$. Bill Fgert from Grould Flectronics for help with the pen motor. We are particularly indebted to Dr. P. Getting for support from his NIH Grant NS17328, and for equipment and space for performing some of the initial experiments. Supported by NSF Grant BNS-8215715 and NIH Grant NS22360 to K.A.S., and NIH Grant NS23216, SCRF Grant NBR 501-5, and IAS Grant ISF 85-3 to A.D.M.

Correspondence should be addressed to Dr. A. D. McClellan, Department of Physiology, University of Iowa, Iowa City, IA 52242.

Copyright (C) 1988 Society for Neuroscience $0270-6474 / 88 / 010133-13 \$ 02.00 / 0$
}

It is now well documented in a variety of animals that the motor patterns underlying many rhythmic motor acts are centrally programmed and can be generated in reasonable detail by "ccntral pattern generators" (CPG) in the absence of sensory feedback (Delcomyn, 1980; Grillner, 1985). Sensory inputs can, however, have strong effects on the motor activity generated by the CPGs. Movement-related sensory feedback can entrain rhythmic motor activity (Wendler, 1974; Andersson et al., 1981; Fitch and Kammer, 1982; Grillner and Wallen, 1982; Robertson and Pearson, 1983). In addition, the CPG can gate sensory inputs to adapt them to the ongoing phase of movement (Duysens, 1977; Andersson et al., 1978). Finally, sensory inputs can initiate rhythmic motor activity (cf. McClellan, 1986).

In vertebrates, the CPGs for rhythmic locomotor activity are distributed in the spinal cord (Grillner, 1985). The in vitro spinal cord of the lamprey, a lower vertebrate, can generate coordinated "fictive" locomotor patterns when excitatory amino acids or their agonists are added to the bath (Cohen and Wallen, 1980; Grillner et al., 1981 b; Brodin et al., 1984; see also Poon, 1980). Rhythmic imposed bending of the notochord/spinal cord, as might occur during locomotion, results in movement-related sensory feedback that can entrain the "fictive" locomotor rhythm (Grillner et al., 1981a). This entrainment persists even when dorsal and ventral roots are cut, suggesting that there are mechanosensitive neurons within the spinal cord that are sensitive to movement of the spinal cord/notochord. The edge cells, located in the lateral white matter (Rovainen, 1974), are mechanosensitive and respond to bending of the spinal cord (Grillner et al., 1984); these neurons have been suggested to mediate the entrainment effect (Grillner et al., 1981a). In the present study, a series of experiments were performed to determine the characteristics of the movement-related feedback in the lamprey spinal cord and the mechanisms by which this feedback is distributed to the spinal CPG motor networks. A preliminary abstract of this work has appeared (McClellan and Sigvardt, 1985).

\section{Materials and Methods}

Adult silver lampreys, Ichthyomyzon unicuspis $(15-25 \mathrm{~cm})$ were anesthetized in tricaine methanosulfonate (MS-222;0.1 mg/liter). In each experiment $(n=17)$, a $30-50$-segment piece of the spinal cord/notochord, usually from the midbody region, was dissected and pinned down in a recording chamber containing a lamprey Ringer's solution (Wickelgren, 1978) maintained at $7-9^{\circ} \mathrm{C}$ by a Peltier cooling apparatus. Locomotor activity was elicited by bath application of $0.25-1.0 \mathrm{~mm}$ D-glutamate or $0.1 \mathrm{mM} N$-methyl D-aspartate (NMDA) and recorded from a rostral and caudal pair of ventral roots by suction electrodes (Fig. $1 A$ ). Either the caudal or rostral free end of the preparation was attached to a pen motor (Gould 867060). The motor was driven by a variableamplitude sine wave generator and imposed a side-to-side movement of the spinal cord, mimicking the movements that would occur during 
A

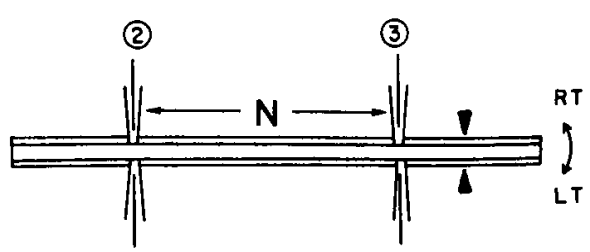

(1)

(4)

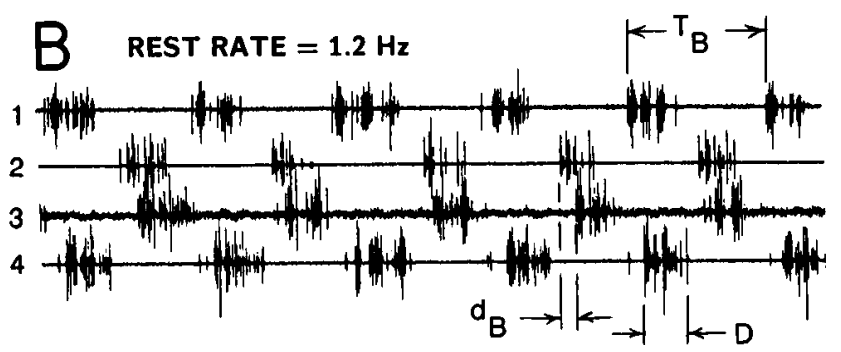

C ENTRAIN (1.65 Hz IMPOSED MOVEMENT)

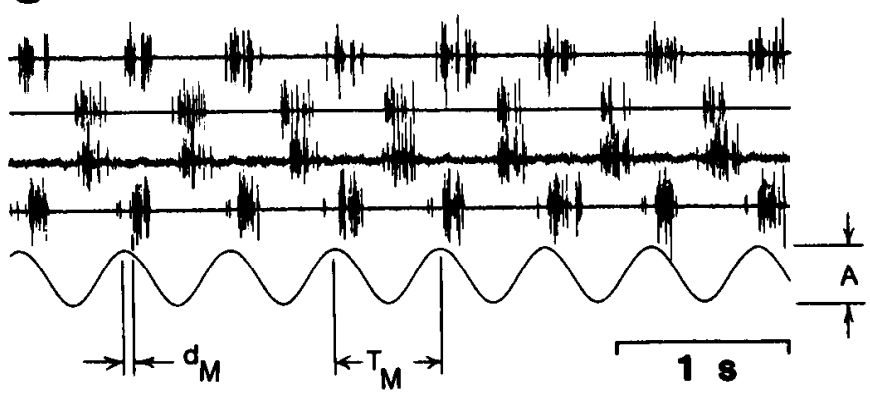

$$
\phi_{B}=\frac{d_{B}}{T_{B}} \div \mathbf{N} \quad \phi_{M}=\frac{d_{M}}{T_{M}}
$$

Figure 1. Summary diagram of entrainment experiments. $A$, In vitro lamprey spinal cord preparation, showing position of suction electrodes (1-4) for recording locomotor activity from vental roots $(\mathrm{N}=10 \mathrm{seg}$ ments). Imposed movements (left) are applied to the free end of the preparation with a pen motor, and the point of bending of the notochord/ spinal cord is indicated by triangles. $B$, With $1.0 \mathrm{~mm}$-glutamate applied to the bath, the spinal cord generates a locomotor rhythm in ventral roots with a resting rate of $1.2 \mathrm{~Hz}$. $C$. With an imposed sinusoidal movement frequency of $1.65 \mathrm{~Hz}(10 \mathrm{~mm}$ peak-to-peak amplitude; upward deflections denote movement to the right), the locomotor rhythm is entrained and is $1: 1$ with the cycles of imposed movement. In this case, the rhythm could be entrained over a range of imposed movement frequencies from 1.1 to $1.65 \mathrm{~Hz}$. For parameter definitions, measured parameters are normally compared within the same cycle. Abbreviations: $A$, peak-to-peak amplitude of imposed movement; $D$, burst duration; $d_{\mathrm{B}}$, burst delay between the onset of bursts in ipsilateral ventral roots; $d_{\mathrm{M}}$, delay between the onset of a ventral root burst and a reference point in the movement trajectory; $T_{\mathrm{B}}$, cycle time or $1 /$ frequency of the locomotor rhythm; $T_{\mathrm{M}}$, movement period or $1 /$ frequency of sinusoidal imposed movement; $\phi_{\mathrm{B}}$, intersegmental phase lag; $\phi_{\mathrm{M}}$, phase of burst within movement cycle.

swimming. Typically, the point of bending was 6 segments $(15 \mathrm{~mm})$ from the free end of the preparation and 3-7 segments $(5-15 \mathrm{~mm})$ from the nearest pair of ventral root electrodes (Fig. 1). The imposed movement amplitudes were $2-10 \mathrm{~mm}$ peak-to-peak, which resulted in angles of bending of $4^{\circ}-20^{\circ}$. Step movements to the left or right were applied by driving the pen motor with pulses. All dorsal roots were cut to prevent peripheral sensory inputs from affecting the spinal motor networks. The preparation could be partitioned with Vaseline-sealed barriers to create independent pools (see Figs. 9, 10). In some experiments, movement- related ascending or descending activity in spinal fascicles was recorded with a low-calcium Ringer's solution (10\% normal calcium with $2 \mathrm{~mm}$ $\mathrm{MnCl}_{2}$ ) in the bath.

Imposed sinusoidal movement of the in vitro spinal cord activates mechanosensitive neurons that can entrain the locomotor pattern at a frequency above and below the rest rate (Grillner et al., 1981a). Entrainment at a particular frequency of imposed movement was considered complete if the phase lag between the motor activity and imposed movement $\left(\phi_{\mathrm{M}}\right.$; Fig. 1) remained constant and 1:1 for more than 30 cycles. Entrainment at frequencies close to the rest rate was checked by comparing the rhythm frequency to the sinusoidal waveform used to produce the imposed movement. The entrainment range was defined as the maximum and minimum imposed movement frequencies over which the motor pattern was entrained. Relative entrainment, in which the motor pattern is only occasionally coupled to the imposed movement, was usually not considered in this study. Neurophysiological data were recorded on an FM tape recorder (Vetter model D) and played back onto an electrostatic recorder (Gould 1000) at sufficient speed to allow measurement of the various parameters listed in Figure 1.

\section{Results}

With D-glutamate added to the bath, the in vitro spinal cord (Fig. $1 A$ ) produces locomotor activity, recorded in ventral roots, with a particular rest frequency. During imposed sinusoidal movements of the free end of the preparation, the locomotor pattern can be entrained above and below the rest frequency. For example, in Figure $1 B$, the rest rate was $1.2 \mathrm{~Hz}$, and the locomotor activity could be entrained from 1.1 to $1.65 \mathrm{~Hz}$ (Fig. 1C). In general, movement to one side tends to inhibit ipsilateral motor activity and produce a ventral root burst on the opposite side. However, the entrainment signal does not simply trigger a contralateral burst, since the phase lag between the burst activity and movement $\left(\phi_{\mathrm{M}}\right)$ varies in a systematic way with changes in cycle time (Grillner et al., 1981a). Entrainment was most effective with moderate lengths of spinal cord (25-40 segments), which typically produce stable motor activity. Longer sections of spinal cord often produce erratic rhythms or partially uncoupled activity in the 2 halves of the preparation; under these conditions, imposed movement could entrain the rhythm in the section of spinal cord nearest the bending and relatively entrain the rhythm in the other half of spinal cord.

\section{Resetting the locomotor rhythm}

Since entrainment can modulate the timing and frequency of the locomotor rhythm, the entrainment signal must have access to the CPG networks. Therefore, one would expect that the entrainment signal could reset the motor pattern. We tested this by applying step movements ( $300 \mathrm{msec}, 4 \mathrm{~mm}$ peak amplitude) to the left or right during different phases of the locomotor rhythm (Fig. $2 A)$. Two general effects were observed $(n=4)$, depending on the phase of the imposed bending. First, step movements to a particular side (e.g., right) during burst activity on that same side (Fig. $2 B$ ) attenuated ipsilateral burst activity (asterisk beside burst in 3). In addition, the entire locomotor pattern was phase-advanced relative to the average timing of the bursts preceding the step (Fig. $2 B$, bars above bursts in 1). Second, step movements to one side (e.g., right) during burst activity on the opposite side (Fig. 2C) augmented contralateral burst activity (asterisk beside burst in trace 4). Furthermore, the locomotor pattern was phase-delayed relative to the timing of burst activity before the imposed movement (Fig. $2 C$; bars above bursts in trace 1). Thus, the expression of either phase advance or phase delay depended on the timing of the step movements relative to the phase of the burst pattern. Step movements to the right and left produced similar but opposite effects. 
A

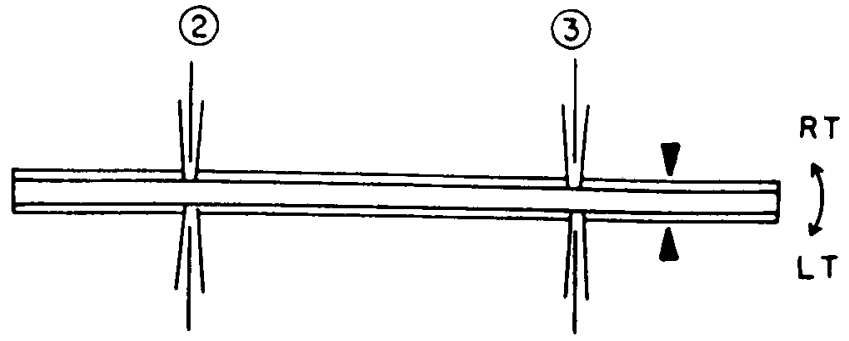

(1)

(4)
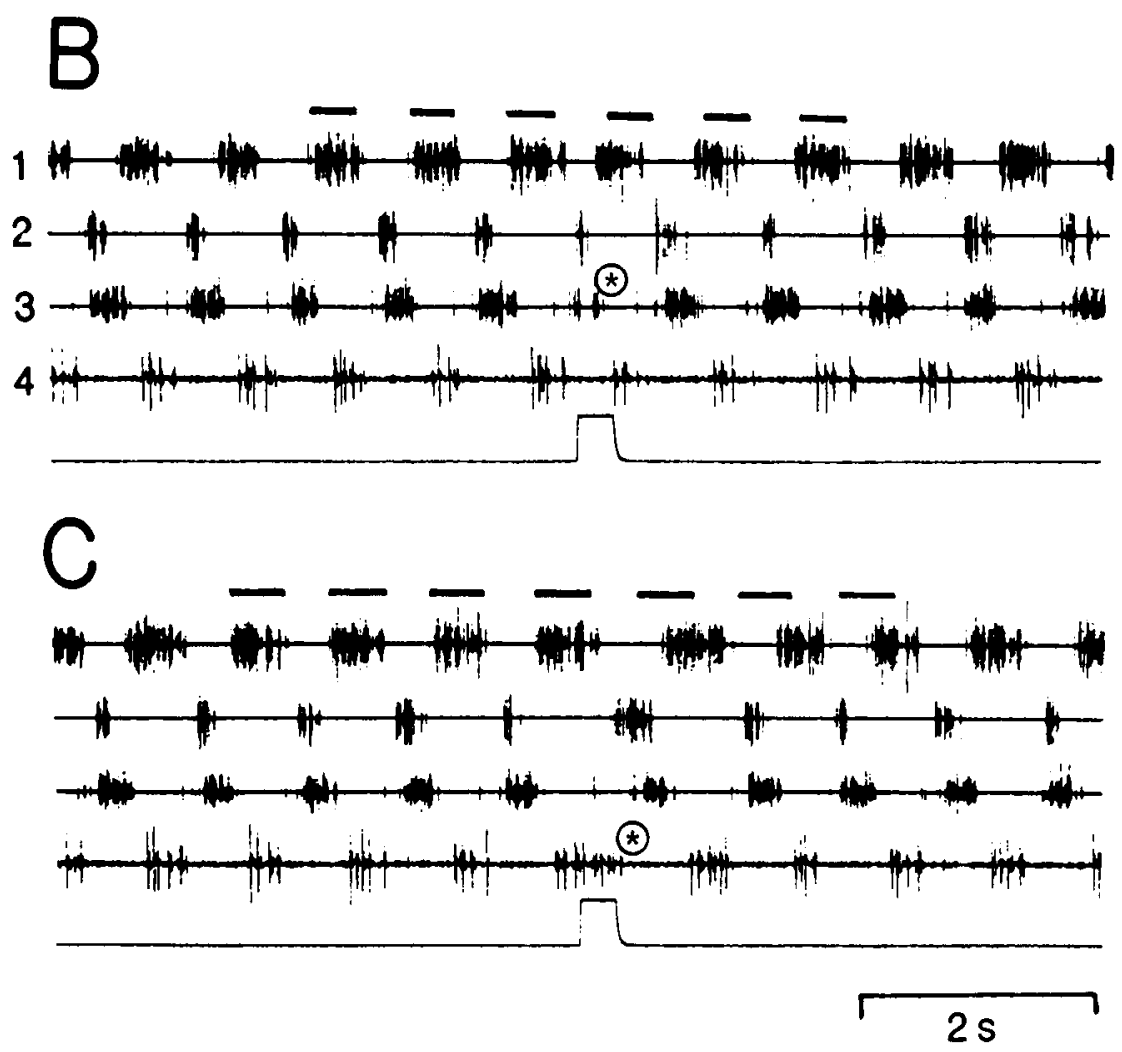

Figure 2. Resetting of the locomotor rhythm by step movements of the caudal end of the notochord/spinal cord. $A$, In vitro spinal cord preparation in which step movements from the rest position ( $4 \mathrm{~mm}, 300 \mathrm{msec}$ ) were applied to the free caudal end of the preparation (left). $(\mathrm{N}=10$ segments.) $B, C$, Effects of applying step movements to the right during different phases of the burst cycle. The average timing of the 3 cycles preceding the step movements are shown as bars above the records. $B$, Single steps to the right (pulse, bottom trace) during burst activity on the same side attenuate bursts in ipsilateral ventral roots 2 and $3(*)$. In addition, the burst pattern in phase-advanced (note bursts in $l$ relative to bars above record). $C$, Single steps to the right (pulse bottom trace) during burst activity on the opposite side of the spinal cord augments bursts in contralateral ventral roots $I$ and $4\left(^{*}\right)$. In this case, the burst pattern is phase-delayed relative to the prepulse average timing of the pattern (bars).

\section{Features of the locomotor pattern during entrainment}

Effects of the amplitude of imposed movement. It is reasonable to expect that the entrainment range would be affected by the intensity of the entrainment signal. As expected, the entrainment range increased asymptotically with increasing movement amplitude when imposed sinusoidal movements were applied to either the caudal or rostral free end of the spinal cord (Fig. 3). The amplitudes ranged from 1 to $8 \mathrm{~mm}$ peak-to-peak and corresponded to an angular movement range of about $2^{\circ}-16^{\circ}$ peak $(n=6)$. In most cases, the motor pattern was entrained over a wider range above than below the rest frequency of the motor pattern (Grillner et al., 1981a). With the largest movements, entrainment occurred at frequencies as much as $40 \%$ above and $10 \%$ below the rest frequency (1.2 Hz; Fig. 3, dotted line). In the example shown here, the motor pattern could not be entrained at the rest rate with movement amplitudes less than 2 $\mathrm{mm}$ peak-to-peak. Instead, at these small movement amplitudes, the entrainment range was above the rest frequency. It was our impression that imposed movements at the rest frequency could have a generalized excitatory effect on the spinal cord, increasing the rhythm frequency above the rest rate. This was often the case when the entrainment was weak and occurred over a relatively narrow range of imposed movement frequencies. Under these conditions, entrainment was only effective in a range above the rest frequency.

Relationship of burst proportion to imposed movement frequency. In the intact, spinalized, and in vitro lamprey spinal cord preparation, the cycle times $\left(T_{\mathrm{B}}\right.$ in Fig. 1) of locomotor activity can vary over a range of at least $0.18-5.0 \mathrm{sec}$ (McClellan and Grillner, 1983; McClellan, 1984; Wallen and Williams, 1984). During changes in the cycle time of the locomotor rhythm, the burst duration remains a constant proportion of the cycle time, such that the burst proportion $\left(D / T_{\mathrm{B}}\right)$ has a mean value of about 0.35 (range, $0.2-0.45$; Wallen and Williams, 1984). In the in vitro spinal cord, imposed sinusoidal movements can also alter the cycle times of the motor pattern, and so it was of interest to examine how this would affect the motor pattern structure. During imposed movement of the caudal end of the spinal cord $(n=6)$, the locomotor rhythm was entrained over a range of frequencies and the burst proportion again remained relatively constant at about 0.35 (horizontal dotted line, Fig. $4 A$ ), which 


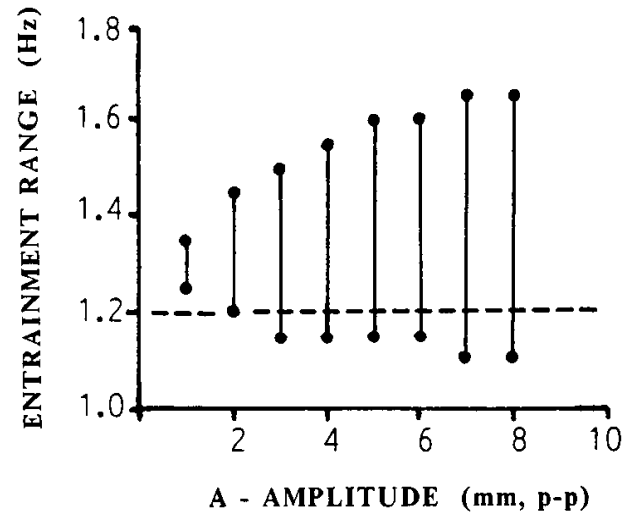

Figure 3. Entrainment ranges (vertical lines) obtained with different amplitudes ( $A$; see Fig. 1) of sinusoidally imposed movement applied to the caudal free end of the spinal cord (see Fig. 1). At movement amplitudes of $1 \mathrm{~mm}$ peak-to-peak, the entrainment range was 1.25 $1.35 \mathrm{~Hz}$ (leftmost vertical line), which was above the rest frequency of $1.2 \mathrm{~Hz}$ (1.0 mM D-glutamate in bath). At the largest movement amplitudes of $8 \mathrm{~mm}$ peak-to-peak, the entrainment range increased to $1.1-$ $1.65 \mathrm{~Hz}$ (rightmost vertical line).

is close to the value for the rest rate (Fig. $4 A$, vertical dotted line). This constant burst proportion was also observed $(n=3)$ during imposed movement of the rostral end of the preparation (Fig. $4 B$ ), although the mean value was about 0.24 in this preparation. This implies that changes in burst duration with cycle time can be controlled locally within the CPG networks and are independent of the means by which the cycle time is varied.

Relationship of intersegmental phase lags during entrainment; imposed movement of the caudal end of the spinal cord. During variations in the cycle time of locomotor activity in the intact, spinalized, and in vitro lamprey spinal cord, the intersegmental phase lag ( $\phi_{\mathrm{B}}$ in Fig. 1) between rostral and caudal ipsilateral ventral root bursts remains constant at about 0.01 (Wallen and Williams, 1984). This value for phase lag translates into one complete undulatory wave over the approximately 100 segments of the lamprey body. Again, it was of interest to examine how changes in the cycle time during entrainment would affect the phase lags. When the cycle time of the locomotor rhythm was varied over the entrainment range, the phase lag did not remain constant. For imposed movement of the free caudal end of the spinal cord (Fig. 5A), the phase lag actually decreased with decreasing cycle time $(n=6)$ below the rest value of 0.012
(Fig. 5A, horizontal dotted line). In this particular example, the phase lag decreased from 0.012 to 0.002 over the entire entrainment range of 620-1010 msec. Interestingly, the phase lag during entrainment at the rest rate was 0.007 , which was less than the natural phase lag of 0.012 at this same cycle time. Recordings from several ipsilateral ventral roots spaced along the length of the spinal cord (Fig. 6A) indicated that imposed movement had a greater effect on the phase lags closer to the point of bending (Fig. $6 B, \phi_{31}$ ) than on those far from the point of bending (Fig. $6 B, \phi_{1-2}$ ). One interpretation of these results is that the mechanosensitive neurons have their strongest effect on CPG networks near the region of bending and less effect on parts of the spinal cord further away.

Relationship of intersegmental phase lags during entrainment; imposed movement of the rostral end of the spinal cord. The phase lags also changed during imposed movement of the rostral end of the preparation (Fig. $5 B$ ) although the effect was more variable and not as dramatic as that obtained from caudal imposed movement. Typically, the phase lags increased for the shortest cycle times of entrainment $(n=3)$ relative to the values at the resting rate (Fig. $5 B$, dotted lines). However, there could be a slight decrease in the phase lags during entrainment at intermediate values of cycle time. The difference in the effects of rostral and caudal imposed movement upon phase lag (Fig. 5) may reflect differences in the ability of the motor networks to adjust to perturbations at various points along the spinal cord (see Discussion).

\section{Extent of ascending and descending projections of mechanosensitive neurons}

Previous work suggested that the entrainment effects in the lamprey spinal cord might be mediated by the edge cells (Grillner et al., 1981a), which are located in the lateral white matter in the spinal cord (Rovainen, 1974) and have been shown to respond to bending of the notochord/spinal cord (Grillner et al., 1984). The edge cells send ascending and descending projections into the lateral fascicles of the spinal cord (Rovainen, 1974; Tang and Selzer, 1979). During imposed movement of the free end of the spinal cord in low-calcium solutions, which block chemical synaptic transmission, unit activity can be recorded in lateral, but not medial, fascicles several segments away from the point of bending (Grillner et al., 1982). We examined the extent of the ascending and descending projections of the mechanosensitive neurons, since this may govern the distri-
Figure 4. Burst proportion $\left(D / T_{\mathrm{B}}\right.$; see Fig. 1) during entrainment at different cycle times during imposed sinusoidal movement at different frequencies of imposed movement $(10 \mathrm{~mm}$ peak-topeak amplitude). Imposed movement of $(A)$ the caudal and $(B)$ the rostral free ends of the in vitro spinal cord preparation. The burst proportion remains relatively constant during changes in cycle time. The asterisk indicates the intersection of the average burst proportion (horizontal dotted lines) at the average cycle time (vertical dotted lines) during the rest rate. Data points are means with SEs given by bars directly above.

\section{A}
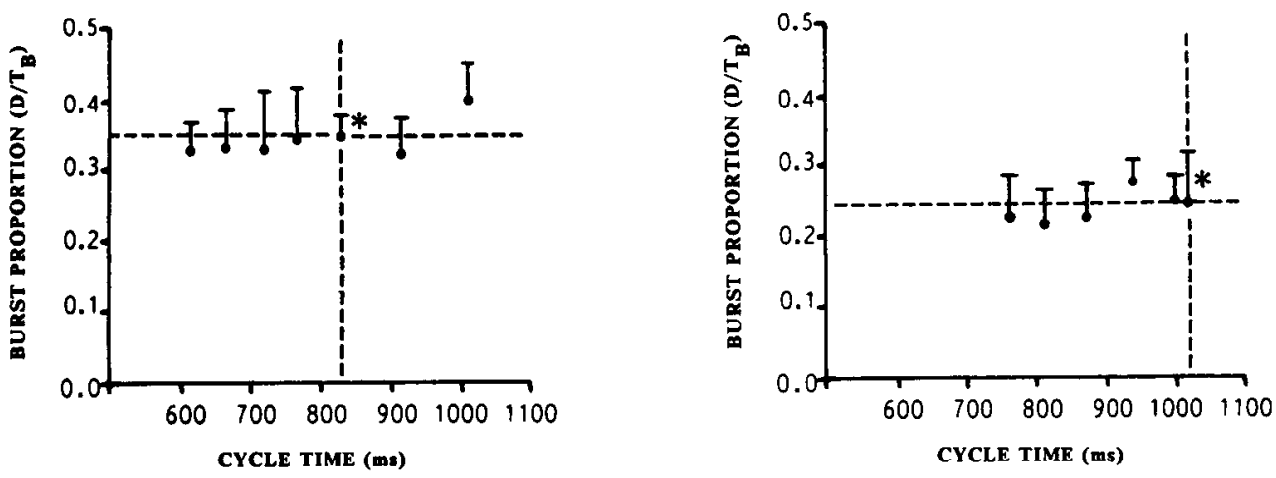

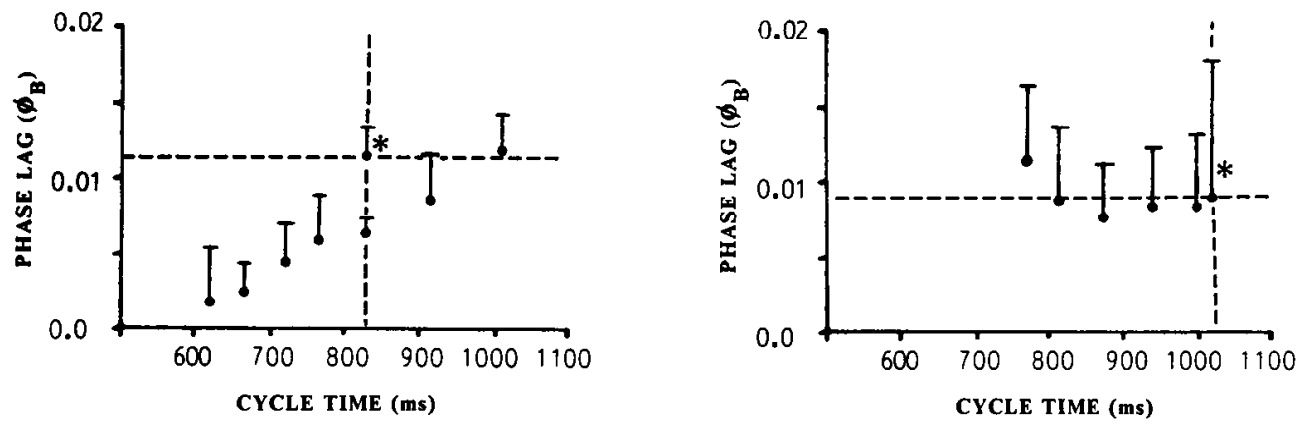

Figure 5. Intersegmental phase lag ( $\phi_{\mathrm{B}}$; see Fig. 1) during entrainment by imposed sinusoidal movement at different cycle times (10 mm peak-topeak amplitude). Horizontal dotted lines indicate phase lags at the rest rates, shown as vertical dotted lines. A, Imposed movement of caudal end of the spinal cord ( $\mathrm{N}=10$ segments; see Fig. 1) resulted in phase lags that decreased from 0.012 to about 0.002 over the entrainment range (620$1010 \mathrm{msec}$ ). $B$, Imposed movement of the rostral end of the spinal cord ( $\mathrm{N}=13$ segments) did not dramatically affect the phase lags, except for the shortest cycle time, where the phase lags increased. The asterisk indicates the intersection of the average phase lag (horizontal dotted lines) at the average cycle time (vertical dotted line) during the rest rate. Data points are means with SEs given by bars directly above.

bution of the entrainment signal to the CPG networks in the spinal cord. The in vitro spinal cord was bathed in a low-calcium Ringer's solution, and recordings were made from the lateral fascicles at various segmental distances from the point of bending (Fig. 7, top). The free end of the notochord/spinal cord was stepped $15^{\circ}-20^{\circ}$ to the left or right. The effects of moving either the caudal or rostral end of the preparation were tested. Bending the caudal part of the preparation $(n=8)$ elicited ascending activity in lateral fascicles as much as 12-24 segments away from the point of bending (Fig. 7). Activity could arise on either the leading or falling edge of the movement trajectory, although some units were also active while the preparation was held at

A

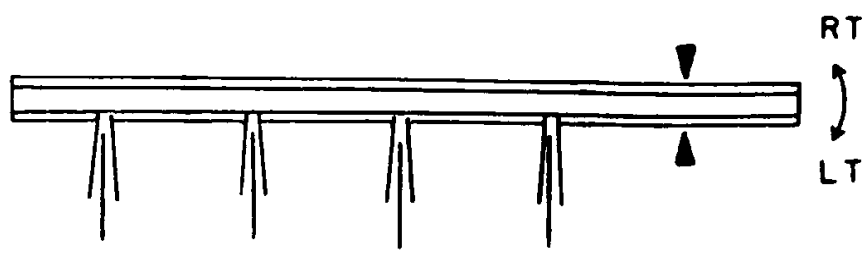

(1)
(2)
RT

LT

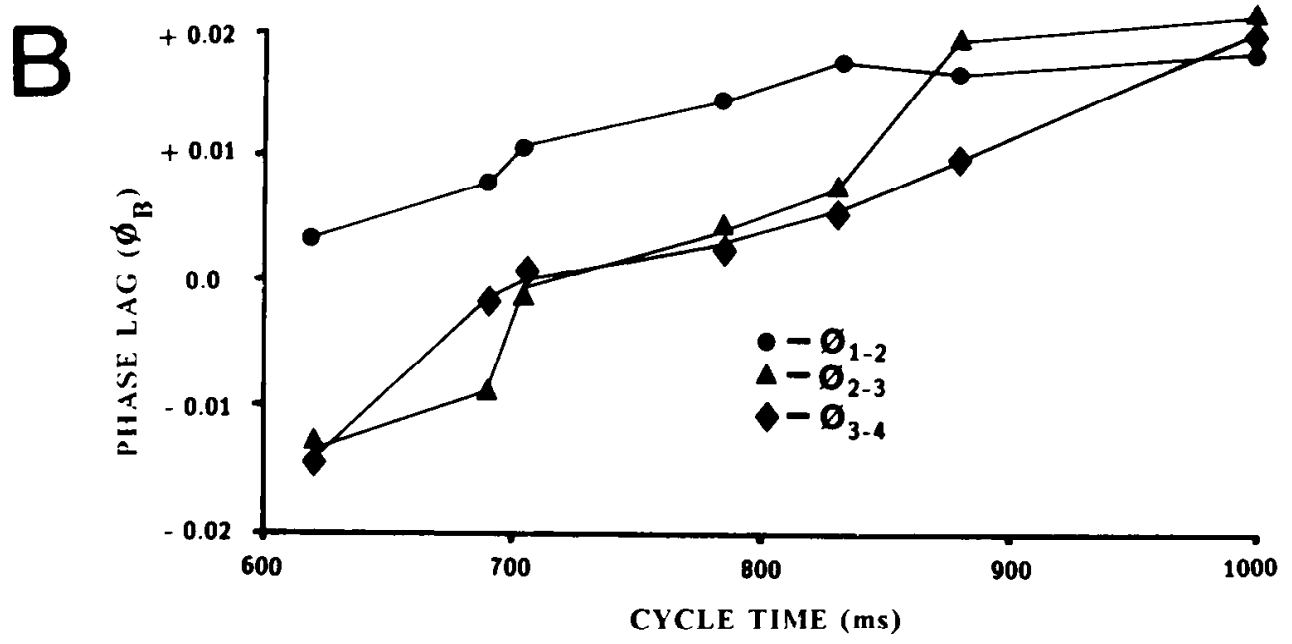

Figure 6. Changes in phase lag during entrainment in different regions of the spinal cord. $A$, In vitro spinal cord preparation showing the site of caudal, imposed movement (right) and the 4 left, ipsilateral ventral root electrodes (1-4) that were evenly spaced between the point of bending and the rostral part of the spinal cord. Separation between electrodes was $8-10$ segments. $B$, Plot of the segmental phase lags during entrainment between the 3 pairs of ventral root electrodes $\left(\phi_{1-2}, \phi_{2-3}, \phi_{3-4}\right)$ over the range of imposed movement cycle times (620-1000 msec). Peak-to-peak amplitude, $10 \mathrm{~mm}$. 

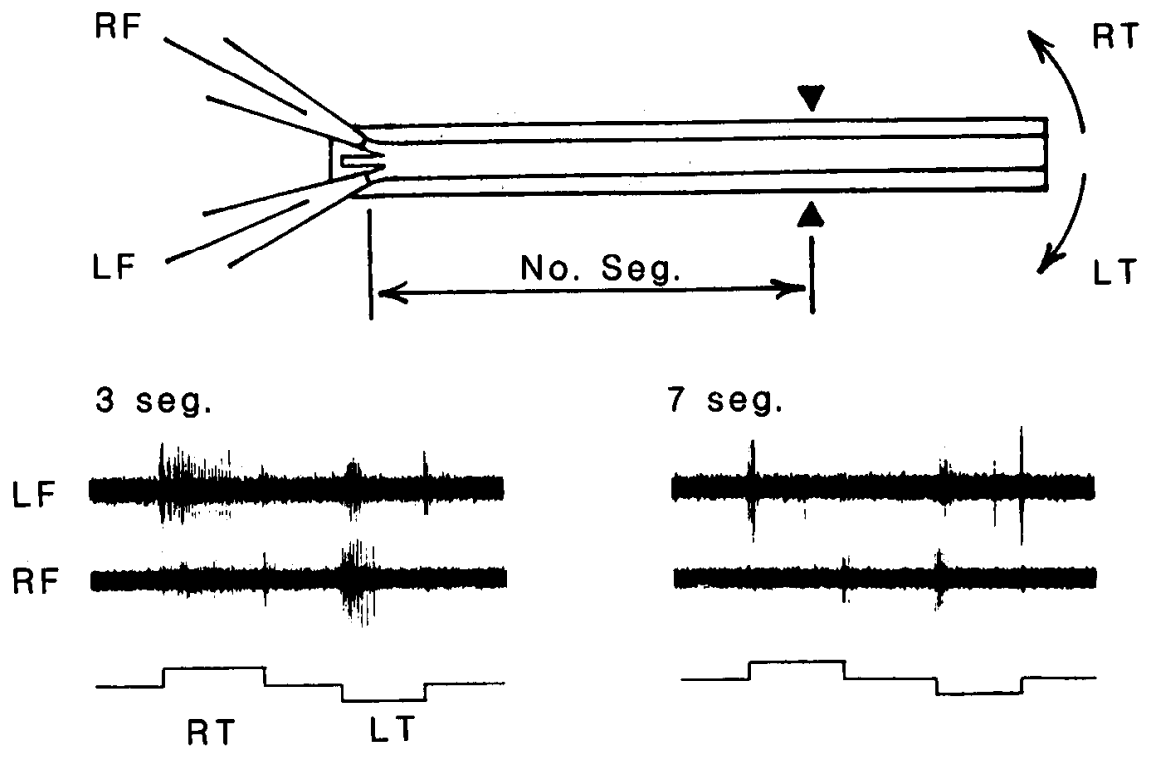

Figure 7. Movement-related activity recorded in lateral spinal fascicles in low-calcium Ringer's solution at increasing distances from the point of bending. Top, Diagram of in vitro spinal cord (rostral lefi) showing right $(R F)$ and left $(L F)$ lateral fascicle recording electrodes and the point of bending (triangles), scparated by various segmental distances. Imposed step movements to the right $(R T)$ or left $(L T)$ are applied to the caudal free end of the preparation. Step movements of the caudal spinal cord to the right or left elicited ascending activity in lateral spinal fascicles many segments away from the point of bending, although the strongest activity occurred closer to the point of imposed movement.
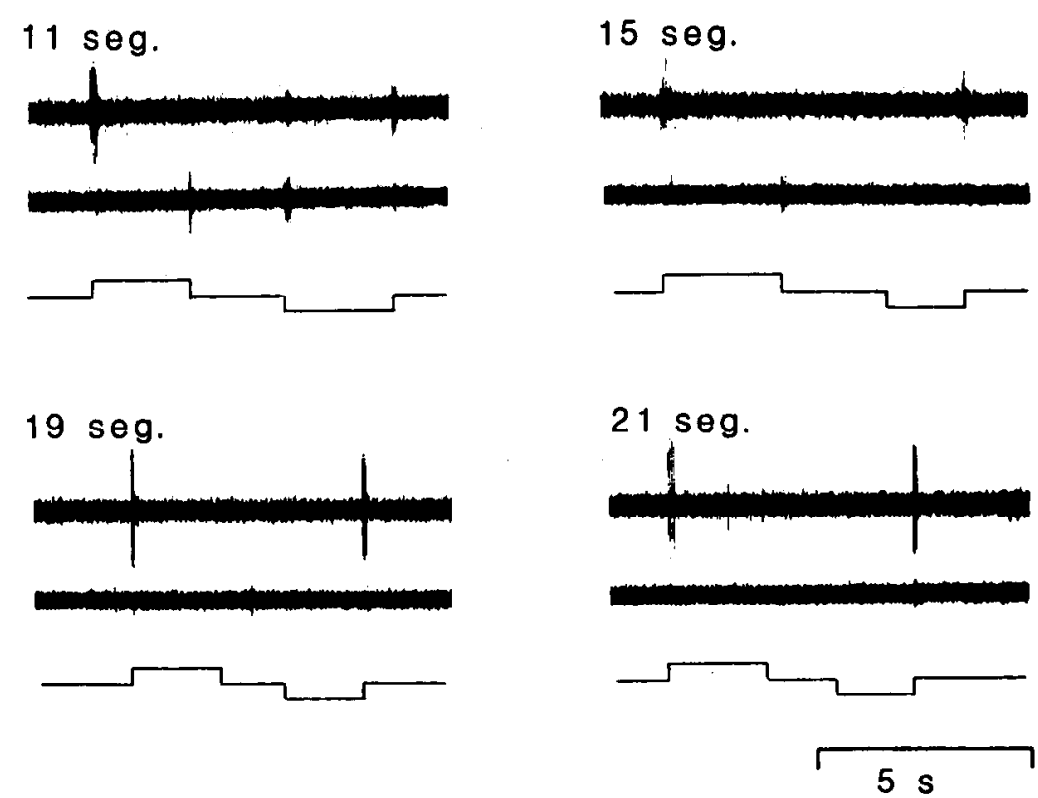

the lateralmust position. Similarly, bending the rostral part of the preparation $(n=3)$ elicited descending activity in lateral fascicles from 5 to 12 segments away from the point of bending. Thus, lateral displacement of the spinal cord elicits both ascending and descending activity in lateral fascicles many segments away from the point of bending; however, the elicited fascicle activity was strongest closer to the point of bending and weaker further away. Since low-calcium solutions block conventional chemical synapses, this activity most likely arises from ascending and descending axons of intraspinal mechanoreceptors near the point of bending.

\section{Manipulations of the spinal cord near the point of bending}

Lesions of the lateral and medial spinal tracts. During rhythmic bending of the spinal cord, intraspinal mechanoreceptors are phasically activated and send a modulatory signal to the CPG networks to entrain the motor pattern. Since the mechanosensitive neurons appear to have moderately long ascending and descending axons, these axons could carry the entrainment sig- nal to the CPG networks. Therefore, we tested the effects of partial spinal lesions placed between the point of bending and the ventral root recordings in order to interrupt possible pathways carrying the entrainment signal to the CPG (Fig. $8 A$ ).

During imposed rhythmic bending of the free caudal part of the preparation $(n=3)$, lesions of the lateral spinal tracts (Fig. $8 \mathrm{~A}$, inset), which would be expected to interrupt the movementrelated activity in lateral fascicles, did not abolish entrainment (Fig. 8, $B, C$ ). In addition, the entrainment range after lesioning the lateral fascicles was not significantly different from the control $(0.95-1.2 \mathrm{~Hz})$. Lesions of the medial tracts also did not significantly affect entrainment. Similar results were obtained when imposed movement was applied to the rostral part of the preparation and the lesions were again placed between the point of bending and the ventral root electrodes. These results suggest that movement-related activity in ascending and descending axons of the mechanoreceptive ncurons is not necessary for entrainment.

Effects of blocking locomotor activity near the point of bending. 
A

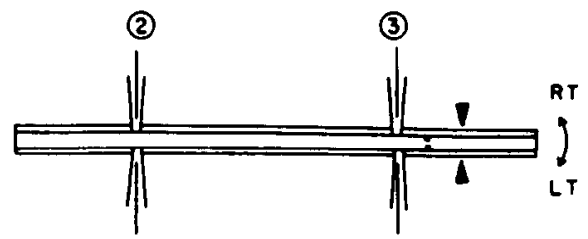

(1)

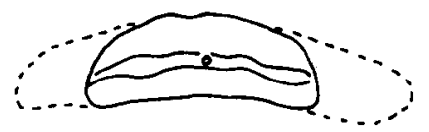

(4)

\section{B}

CONTROL (1.2 Hz; REST $=0.95 \mathrm{~Hz})$
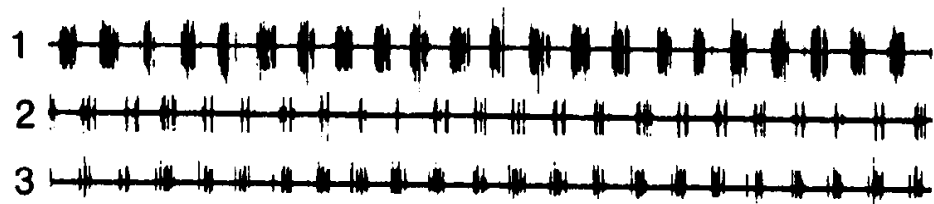

4
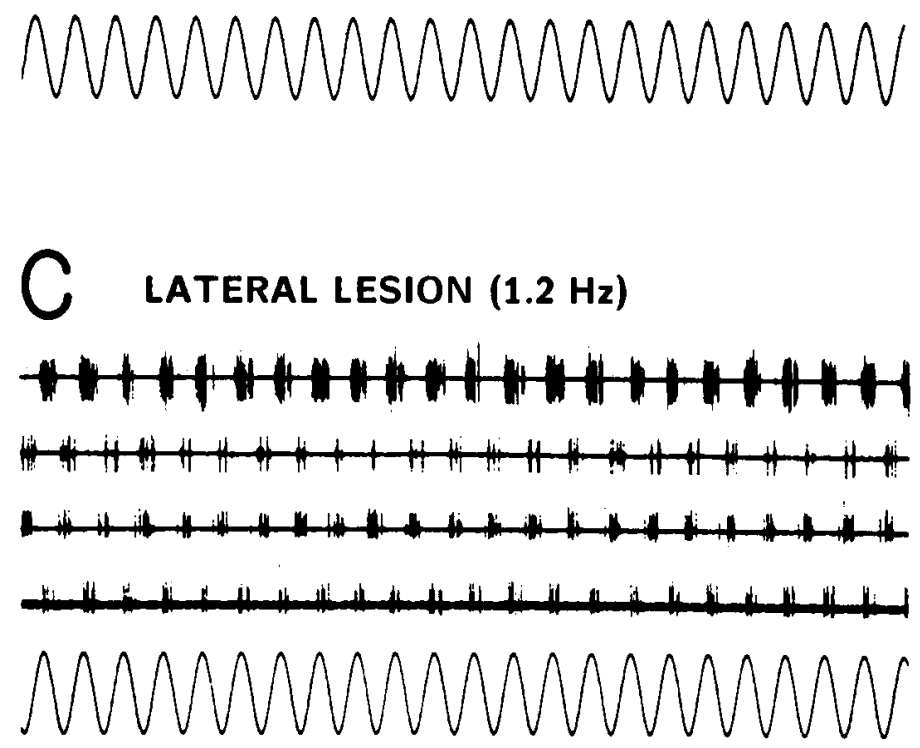

$5 \mathrm{~s}$

Figure 8. Effects on entrainment of lesioning the lateral spinal tracts. $A$, In vitro spinal cord preparation (rostral left), showing ventral root recording electrodes ( $1-4 ; \mathrm{N}=9$ segments) and point of bending (triangles). Lesions of lateral fascicles (inset) were made 3 segments rostral to the point of bending in order to interrupt ascending activity from mechanoreceptive neurons (see Fig. 7). $B$, In the control, imposed movement at $1.2 \mathrm{~Hz}$ entrained the locomotor rhythm in a $1: 1$ fashion (rest rate, $0.95 \mathrm{~Hz}$ ). $C$, After lesions of the lateral tracts, entrainment at $1.2 \mathrm{~Hz}$ still occurred $1: 1$.
If ascending or descending activity in the lateral fascicles is not necessary in order to entrain the motor pattern, perhaps the mechanoreceptors exert their effects locally on the motor networks. To test this possibility, a sealed barrier was placed between the point of bending and the ventral root recordings to create 2 independent pools (Figs. 9A, 10A). The part of the preparation containing ventral root electrodes was bathed in Ringer's solution containing D-glutamate in order to elicit locomotor activity. The part of the preparation containing the point of bending could then be bathed in different solutions in order to test the organization of the entrainment system. During imposed movement of the caudal free end of the spinal cord (10 mm peak-to-peak amplitude), with D-glutamate Ringer's in both pools, entrainment of the motor pattern occurred as before
(Figs. 9B, 10B). The locomotor activity in the region of bending was then blocked by applying either normal or low-calcium Ringer's to the pool where the imposed movement was applied $(n=5)$. The general effect of either of these 2 manipulations was to abolish 1:1 entrainment. For example, with Ringer's in the caudal pool (Fig. 9A), rhythmic imposed movement no longer entrained the locomotor rhythm even at frequencies just above or just below the rest rate (Fig. 9, $C, D$ ).

In one particular preparation (Fig. 10, $A, B$ ), there was still some weak relative entrainment at an imposed movement of $1.25 \mathrm{~Hz}$ with low-calcium Ringer's in the caudal pool (Fig. 10C). In this case, the locomotor pattern (rest rate, $1.12 \mathrm{~Hz}$ ) would occasionally "lock onto" the imposed movement for a few cycles (Fig. 10D); however, this effect was abolished when the imposed 
Figure 9. Effects on entrainment of blocking the locomotor rhythm in the region of bending. $A$, In vitro spinal cord preparation, showing a Vaseline-sealed barrier (vertical line) separating the rostral pool, where ventral root electrodes ( $I-4 ; \mathrm{N}=8$ segments) were located, from the caudal pool, where the imposed movement and point of bending were located. $B$, With $0.5 \mathrm{~mm}$ D-glutamate Ringer's in both pools, imposed movement at $1.55 \mathrm{~Hz}(10 \mathrm{~mm}$ peak-to-peak amplitude) entrained the locomotor rhythm 1:1 (rest rate, $1.15 \mathrm{~Hz}$ ). $C, D$, Normal Ringer's in the caudal pool; D-glutamate Ringer's in the rostral pool to produce locomotor activity (rest rate, $1.2 \mathrm{~Hz}$ ). Imposed movement at 1.15 $\mathrm{Hz}$, just below the rest rate, or at 1.3 $\mathrm{Hz}$, just above the rest rate, did not entrain the locomotor rhythm. Ascending axons of mechanoreceptive neurons in the region of bending would be expected to conduct movement-related activity into the rostral part of the spinal cord, where locomotor activity was generated (see Fig. 7).
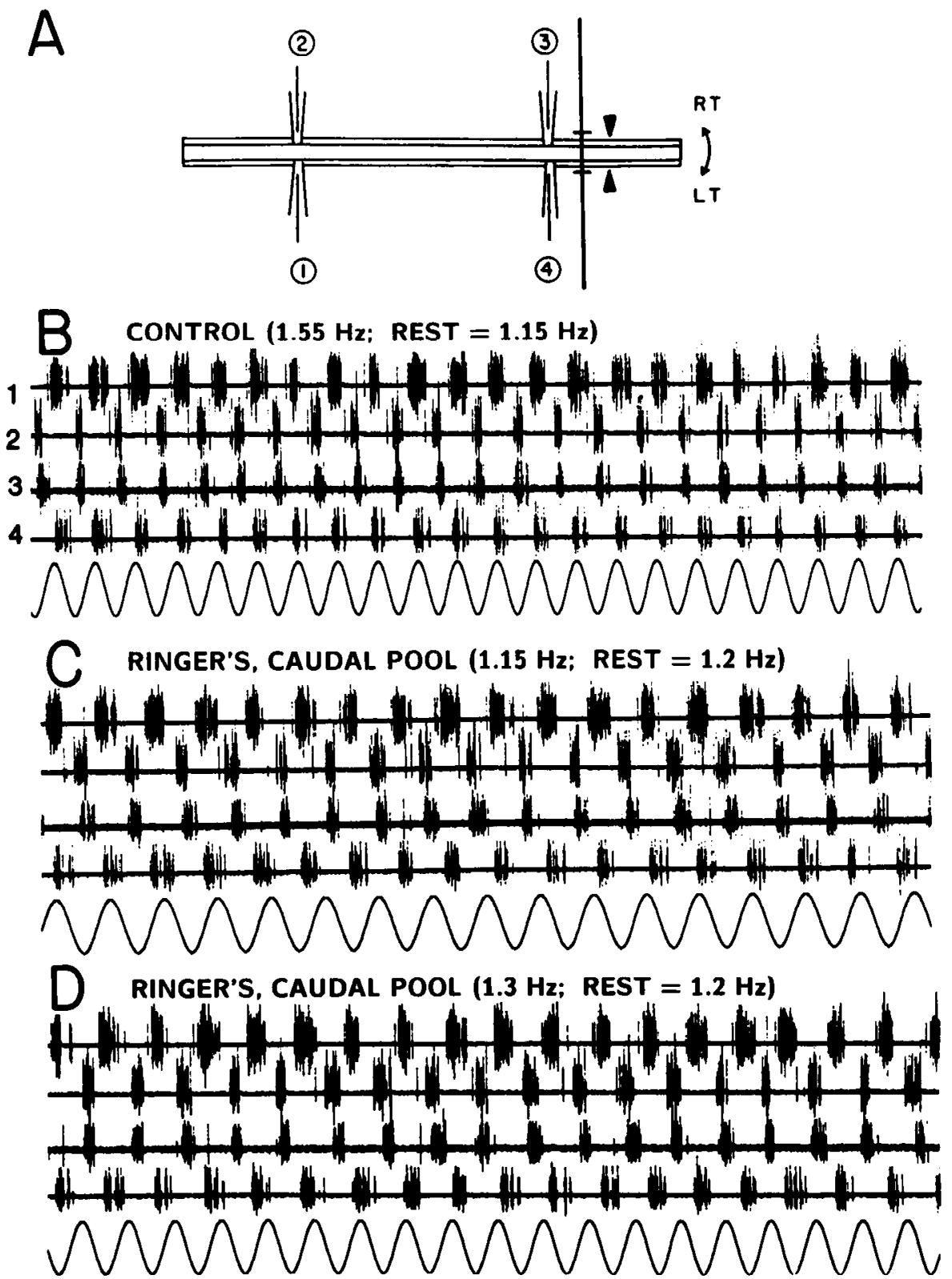

movement frequency was increased to $1.3 \mathrm{~Hz}$. Thus, this rather weak coupling occurred at imposed movement frequencies of only $8 \%$ above the rest rate, compared to the control situation, in which $1: 1$ entrainment occurred at $35 \%$ above the rest rate.

Therefore, blocking the locomotor rhythm in the region of bending abolishes (or at least severely reduces) entrainment of the pattern in more rostral parts of the spinal cord. This disruption of entrainment occurs despite the fact that movementrelated activity in the lateral fascicles is still reaching the rostral spinal cord, where locomotor activity is generated. Similar results were obtained when the spinal cord was reversed and imposed movement was applied to the rostral free end of the preparation. These results and those described above indicate that movement-related activity in the ascending and descending axons of mechanosensitive neurons is neither necessary (Fig. 8) nor sufficient (Figs. 9, 10) to fully entrain the locomotor rhythm. Therefore, the mechanosensitive neurons appear to entrain the locomotor pattern predominantly by affecting the CPG networks locally. This timing information could then be distributed to the remaining parts of the spinal cord through the coordinating system.

Location of the mechanoreceptor neurons. All available evidence suggests that the mechanosensitive neurons responsible for producing entrainment are located in the lateral margins of the spinal cord in the white matter (Grillner et al., 1984). This possibility was examined directly by testing the effects on the entrainment of lesions of the lateral margins of the spinal cord spanning the region of bending (Fig. $11 A$ ). In the control (Fig. $11 B$ ), imposed movement of the caudal free end of the preparation entrained the locomotor rhythm. Stripping away the leftmost lateral margin of the spinal cord for 3 segments above and below the point of bending did not abolish entrainment, but reduced the entrainment range (Fig. $11 C$ ). When both the leftand rightmost lateral margins of the cord encompassing the 

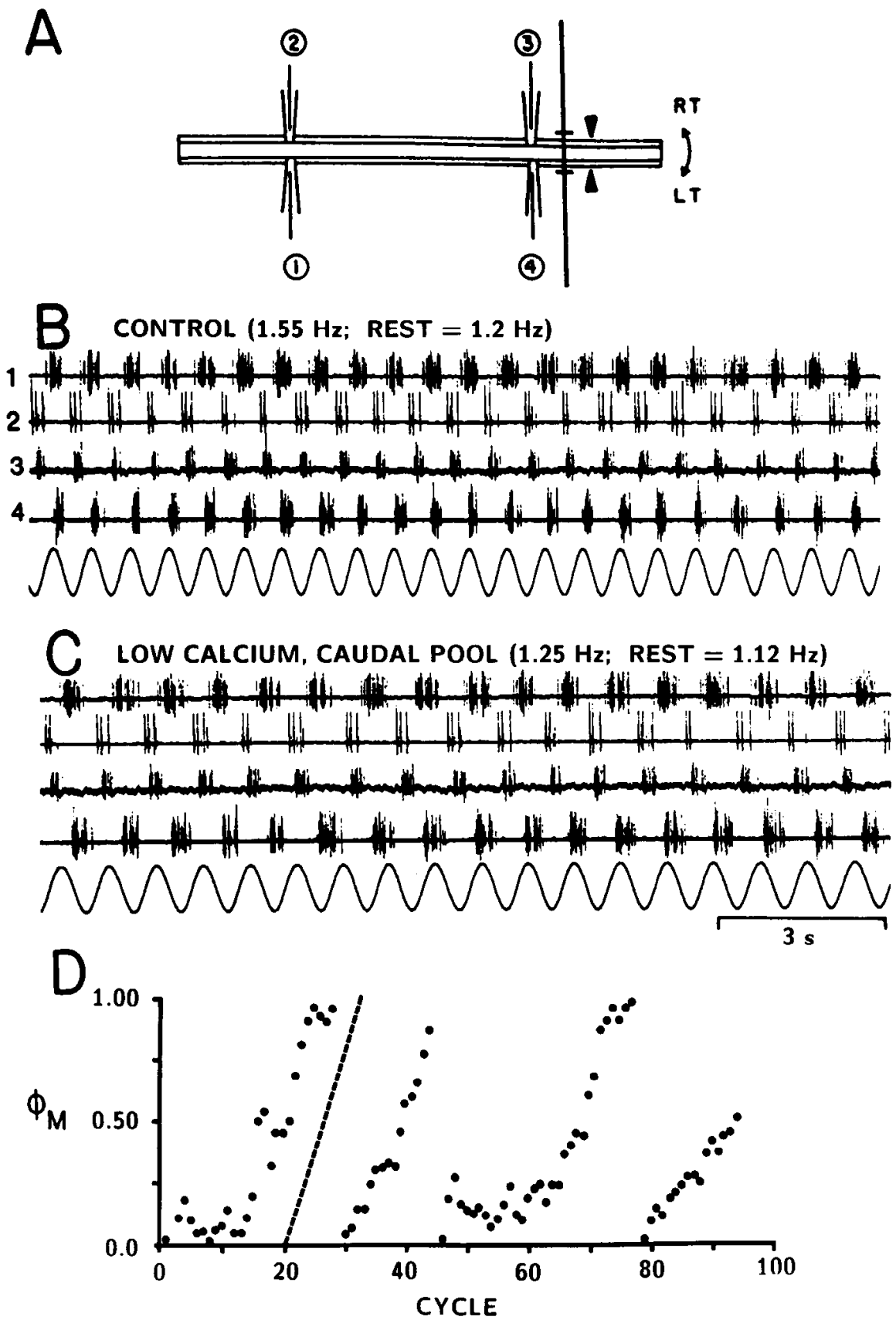

Figure 10. Effects on entrainment of blocking synaptic activity in the region of bending. $A$, In vitro spinal cord preparation as described in Figure $9 A .(\mathrm{N}=$ 10 segments.) $B$, With $1.0 \mathrm{~mm}$ D-glutamate Ringer's in both pools, imposed movement at $1.55 \mathrm{~Hz}$ entrained the locomotor rhythm $1: 1$ (rest rate, $1.2 \mathrm{~Hz}$ ). $C, D$, Low-calcium Ringer's ( $10 \%$ normal calcium) in the caudal pool, to block the rhythm and synaptic transmission in the region of bending, and D-glutamate Ringer's in the rostral pool to produce locomotor activity (rest rate, 1.12 $\mathrm{Hz}$ ). $C$, Imposed movement at $1.25 \mathrm{~Hz}$ resulted in weak relative entrainment, in which the locomotor pattern would occasionally "lock onto" the imposed movement (see $D$ ). $D$, Cycle-by-cycle phase $\left(\phi_{\mathrm{M}}\right)$ of the burst activity relative to the imposed movement. Note that the burst activity occasionally locks onto the imposed movement (cycles $0-10$ and 50-60) but at other times the two are uncorrelated. Slope of dotted line $\left[T_{\mathrm{b}} /\right.$ $\left.\left(T_{\mathrm{B}}-T_{\mathrm{M}}\right)\right]$ indicates the theoretical rate at which the burst activity should slide by the imposed movement if the 2 were completely uncorrelated.

region of bending were removed ( $n=3$; Fig. $11 \mathrm{~A}$, inset), entrainment was completely abolished (Fig. 11D). Therefore, entrainment is most likely mediated by mechanosensitive cells with transduction processes (presumably dendrites) in the lateral margin of the spinal cord.

\section{Discussion}

Although the lamprey spinal cord contains a CPG for generating the basic locomotor pattern, movement-related sensory feedback has a profound effect on the timing and frequency of the rhythm. Rhythmic activation of intraspinal mechanoreceptors during locomotor rhythms can entrain the pattern over a range of frequencies that spans the rest rate. During locomotion, the body and spinal cord bend approximately to the same degree, so transduction of body movements can presumably be detected by mechanosensitive neurons that are intrinsic to the spinal cord. Mechanoreceptors intrinsic to the nervous system have been described in the crayfish (Grobstein, 1973), but not in vertebrates other than the lamprey, although there is some evidence to suggest that they exist (Grillner et al., 1982, 1984; Schroeder and Richardson, 1985).

Interestingly, the entrainment range is asymmetrical; entrainment is more effective above than below the rest rate. This suggests that the movement-related sensory signals are more effective in cycle-by-cycle phase advancement of the rhythm, increasing the rhythm frequency, than in phase-delaying cycles in the rhythm. This could be tested by measuring the phase response curves. Alternatively, or in addition, the direct en- 
A

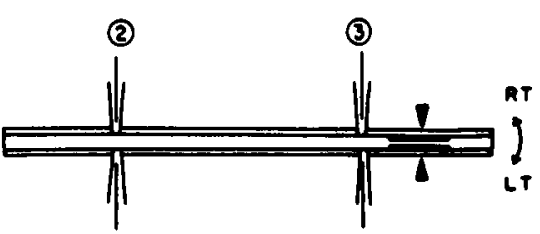

(1)
()
Figure 11. Effects on entrainment of lesioning the lateral margins of the spinal cord in the region of bending. $A$, In vitro spinal cord preparation showing site of lesions of lateral margins (inset) of spinal cord spanning 3 segments above and below the point of bending (triangles). ( $\mathrm{N}=10$ segments.) $B$, In the control, imposed sinusoidal movement at $1.35 \mathrm{~Hz}(10 \mathrm{~mm}$ peak-to-peak amplitude) entrained the locomotor rhythm 1:1. Entrainment range, $0.75-1.35 \mathrm{~Hz}$; rest rate, $1.2 \mathrm{~Hz}$. $C$, After lesioning of the left lateral margin of the spinal cord in the region of bending, imposed movement at $1.3 \mathrm{~Hz}$ still entrained the rhythm 1:1. Entrainment range, 1.1-1.3 $\mathrm{Hz}$; rest rate, $1.0 \mathrm{~Hz}$. $D$, After bilateral lesions of the lateral margins of the spinal cord in the region of bending, locomotor rhythm could not be entrained by the imposed movement even at frequencies close to the rest rate.
CONTROL (1.35 Hz; REST $=1.2 \mathrm{~Hz})$

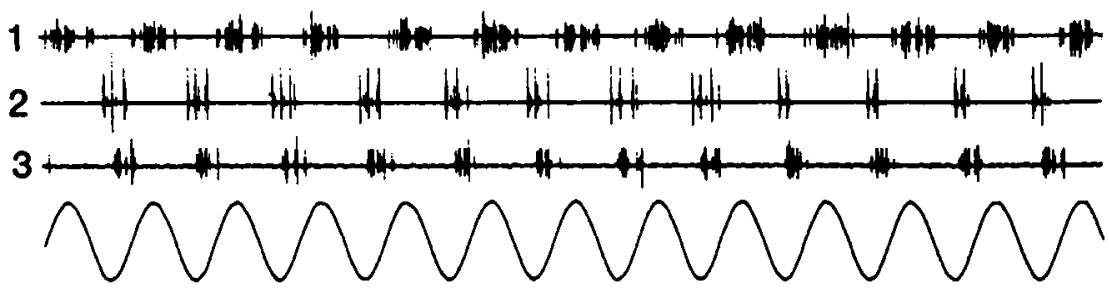

C heft lesion (1.3 Hz)
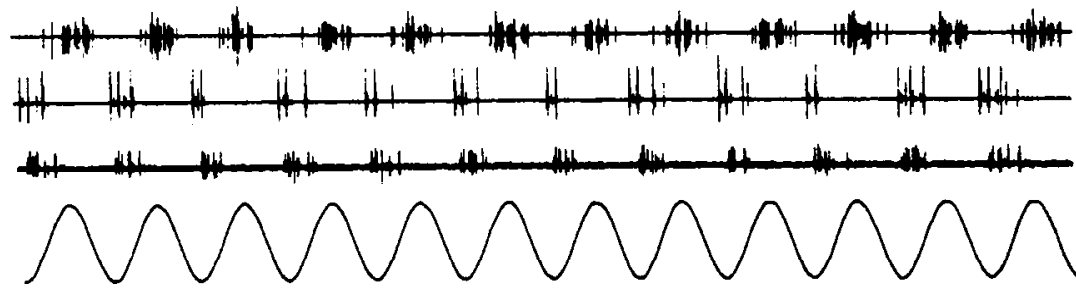

$D$ BILATERAL LESION $(1.25 \mathrm{~Hz})$

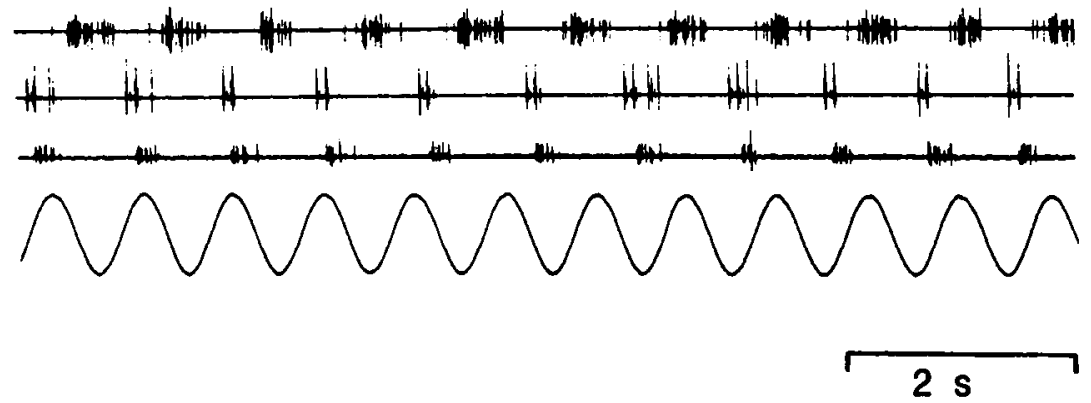

trainment of local oscillators could be affected in an asymmetrical fashion by the nature of the coupling to more distant unit oscillators in the spinal cord.

\section{Theoretical implications for local entrainment of CPGs}

Locomotor activity can be generated by sections of the lamprey spinal cord containing as few as 1.5-2.0 segments (Cohen and Wallen, 1980; Grillner and Wallen, 1984). Therefore, it has been hypothesized that the CPG networks consist of a series of unit oscillators that are distributed in the spinal cord and coupled by coordinating fibers (Grillner, 1974; Cohen and Wallen, 1980). The isolated lamprey spinal cord usually produces locomotor rhythms with positive, rostrocaudal phase lags. The final phase lag will depend on at least 2 factors: (1) the differences in intrinsic frequencies of the unit oscillators, and (2) the direction and strength of coupling between the unit oscillators. Distributed pattern generators containing pacemaker unit oscillators that entrain the motor activity in other oscillators with slower rhythm frequencies have been proposed for swimmeret rhythms in crustaceans (Ikeda and Wiersma, 1964; Stein, 1976), gastric rhythms in crayfish (Robertson and Moulins, 1981; Eisen and Marder, 1984), leech heartbeat system (Peterson and Calabrese, 1982), and walking in lobster (Ayers and Davis, 1977).

The data presented in this paper (Figs. 8-11) strongly suggest 
that intraspinal mechanoreceptors in the lateralmost margins of the lamprey spinal cord entrain the CPG networks locally. Thus, the entrainment signal can be thought of as a "driving oscillator" (Pinsker and Ayers, 1983) that entrains nearby local unit oscillators, which then distribute this timing information to other parts of the motor networks through the coordinating system. If one considers only the effects of intrinsic unit oscillator frequencies on the coordination of motor patterns in the in vitro spinal cord preparation, movement-related feedback onto local oscillators would be expected to produce the following relationship between frequency and phase lag: Imposed movement of the caudal end of the spinal cord above the rest rate will phase advance each burst in nearby oscillators, thereby increasing their frequency. Thesc cyclc-by-cycle phase advances would then reduce the phase lags between the rostral and caudal unit oscillators (Figs. 5A,6). Similar reductions or even reversals of phase lag can occur when the caudal spinal oscillators are made to burst at a higher intrinsic rate than that of the rostral oscillators, such as when the concentration of excitatory amino acids is higher in caudal spinal cord pools than in rostral pools (Rovainen, 1985). In contrast, imposed movement of the caudal end of the spinal cord below the rest rate will phase delay the local unit oscillators and result in an increase in the phase lag (Fig. 5A). The above changes in phase lag indicate that coupling of unit spinal oscillators (i.e., via the intersegmental coordinating system) in the caudorostral direction is present, although this coupling appears to be relatively weak.

Imposed movement of the rostal free end of the spinal cord above the rest rate will tend to phase advance the rostral unit oscillators, resulting in an increase in the intersegmental phase lag (Fig. $5 B$ ) relative to the usual value of about 0.01 . However, this effect is weak and is only seen during entrainment at the shortest cycle times (Fig. $5 B$ ). One explanation for this weak effect is that the coupling of unit spinal oscillators in the rostrocaudal direction is rather strong and does not allow substantial increases in the phase lags of the motor pattern that would produce multiple waves of body undulations.

If the intraspinal mechanoreceptors act locally on CPG networks near the point of bending (Fig. 6), what is the purpose of activity carried in the ascending or descending axons of the mechanosensitive neurons? One possibility is that this activity carries timing and/or amplitude information to motor networks in other parts of the spinal cord, but cannot entrain the rhythm by itself. In addition, some of the ascending axonal activity could reach the brain stem, where it could be used to make adjustments in the descending control of locomotor activity.

\section{Role of movement-related sensory feedback during locomotion}

In the experimental paradigm used here, it was possible to provide evidence that the intraspinal mechanosensitive neurons were entraining the spinal CPG networks locally because imposed bending was applied to a restricted part of the spinal cord. This experimental situation resulted in changes in phase lags, particularly for imposed movement of the caudal end of preparations (Fig. 5A) that differed from the normal value of 0.01 observed during actual locomotion (Wallen and Williams, 1984). However, during actual locomotion, mechanosensitive neurons along the entire spinal cord would be activated by the undulatory movements of the body. Therefore, each of the mechanosensitive neurons along the spinal cord would affect the nearby unit oscillators, and this might then result in normal values of intersegmental phase lags.
How would the intraspinal mechanoreceptors be expected to contribute during actual locomotion? First, when locomotor activity and resultant movement are closely coupled, intraspinal mechanoreceptors would still be activated, but might play a relatively minor role in modulating locomotor activity. However, when there is a mismatch between the timing of locomotor activity and the resultant undulatory movement, one would expect the input from intraspinal mechanoreceptors to make corrections in the rhythm by phase-advancing or phase-delaying the burst activity. Mismatches will undoubtedly occur during rapid changes in movement or perturbations, such as unexpected water currents, prey capture, turning, accelerations and decelerations, and swimming against an increased drag. Second, movement-related sensory feedback might be used to correct for irregularities in the central generated locomotor rhythm. The in vitro spinal cord occasionally produces an irregular or erratic rhythm that becomes much better coordinated during entrainment. Third, movement-related feedback might also compensate for rapid growth during development that could alter the match of motor activity and resultant movement.

During undulatory locomotion in a natural environment, intraspinal mechanoreceptors are only one of the modulatory influences on the spinal CPG networks. Inputs from sensory neurons with peripheral axons and transduction processes, such as spinal dorsal cells (Martin and Wickelgren, 1971) and dorsal root ganglion cells, as well as vision, olfaction, vestibular, the lateral line system, and cutaneous inputs, could be used to modify the ongoing locomotor pattern. Finally, descending command systems in the brain stem (McClellan, 1986) can adjust the frequency and dircetion of locomotion to suit the needs of the animal.

\section{Do the mechanosensitive edge cells entrain the locomotor rhythm?}

All available evidence indicates that the intraspinal mechanoreceptor neurons are located in the lateral margins of the spinal cord (see Fig. 11) and have moderately long ascending and descending axons in lateral spinal tracts. The edge cells are also located in the lateral aspects of the spinal cord (Rovainen, 1974) and have axons with roughly similar distributions (Tang and Selzer, 1979). Recent evidence indicates that these cells are mechanosensitive and respond to small stretches (less than 5\%) of the lateral edges of the spinal cord (Grillner et al., 1984). Thus, the edge cells are excellent candidates for transducing bending of the spinal cord (and body) and providing movementrelated sensory feedback to the CPG networks. If the edge cells are mediating the entrainment effects observed in the lamprey spinal cord, then they must have direct or indirect inputs to the CPG networks

The edge cells have predominantly ipsilateral and contralateral ascending axons (Rovainen, 1974). About $20 \%$ of the cells have descending axons, and some cells have both descending and ascending axons (Tang and Selzer, 1979). The ascending axons of edge cells have been reported to project for a maximum of about $3.7 \mathrm{~cm}$ into a river lamprey spinal cord measuring 18.5 $\mathrm{cm}$ (Rovainen, 1974; Tang and Selzer, 1979). We have used adult silver lampreys (typically $25-35 \mathrm{~cm}$ total length), which are considerably longer than the animals used in the above studies. In our study, the ascending axons of mechanoreceptor neurons can extend for as much as $3.5-7.0 \mathrm{~cm}$ (12-24 segments), while the descending axons of mechanoreceptor neurons appear to extend for shorter distances (5-12 segments). Finally, neurons 
located in the lateral aspects of the spinal cord are said to be somewhat heterogenous (Selzer, 1979), and it is not clear if all of these neurons can be considered edge cells.

\section{Comparison to entrainment in other systems}

Entrainment of locomotor activity has also been examined in a series of related studies in the dogfish (Grillner and Wallen, 1982), in which imposed cyclic movement of the tail can entrain the "fictive" locomotor pattern. However, entrainment in the dogfish appears to be much stronger than that in the lamprey, and, more significantly, entrainment does not significantly alter the normal rostrocaudal phase lags. One possible explanation for these different effects on phase lag is that sinusoidal oscillations of the tail in the dogfish may mimic locomotor movements more closely than does the restricted bending that occurs during sinusoidal movements of the lamprey spinal cord (Fig. $1 A$ ). Nonetheless, entrainment of locomotor activity in the dogfish and lamprey is qualitatively similar and may use some of the same mechanisms.

Entrainment of rhythmic motor activity by movement-related sensory feedback has been described in a number of other systems, including insect flight (Wendler, 1974; Möhl and Nachtigall, 1978; Pearson et al., 1983; Robertson and Pearson, 1983), ventilatory rhythms in arthropod larvae (Fitch and Kammer, 1982), and cat locomotion (Andersson et al., 1978). In addition, evoked excitatory and inhibitory synaptic potentials have been used to entrain the rhythmic motor activity in small neural networks (Ayers and Selverston, 1979). The basic principles of entrainment can also be important for coordination; 2 or more oscillators can be coupled together and mutually entrain each other (Stein, 1971, 1976; Bacon and Tyrer, 1979; Young and Coyer, 1979). Therefore, the principles of entrainment appear to be a general mechanism of motor systems.

Several studies have investigated the effects of artificial or synaptic membrane polarization on controlling the timing of firing in neurons with endogenous membrane properties, such as pacemaker or bursting cells. In both crayfish and the marine mollusk Aplysia, repetitive IPSPs or EPSPs can alter the firing in pacemaker neurons and produce stable, time-locked firing patterns that are entrained to the input (Perkel et al., 1964; Pinsker, 1977a, b). Finally, in the presence of NMDA (with tetrodotoxin in the bath), the membrane potentials of neurons in the lamprey spinal cord undergo oscillations, which can be reset by short, polarizing current pulses (Grillner and Wallen, 1985; Sigvardt et al., 1985). It would be very interesting to determine whether mechanosensitive neurons in the lamprey spinal cord could also affect the timing of activity in these neurons with oscillatory properties.

\section{Conclusion}

Intraspinal mechanoreceptors within the lamprey spinal cord have access to the CPG networks, and can reset, as well as entrain, the locomotor rhythm. Several of the results discussed here suggest that the mechanoreceptors have their strongest effects on spinal motor networks only within the segments nearest the mechanical transduction site. Thus, the mechanosensitivc neurons that are distributed along the length of the spinal cord would each effect nearby parts of the CPG. This scheme forms the basis of a powerful sensory feedback system for modulating and coordinating the spinal locomotor pattern in the lamprey.

\section{References}

Andersson, O., H. Forssberg, S. Grillner, and M. Lindquist (1978) Phasic gain control of the transmission in cutaneous reflex pathways to motoneurons during "fictive" locomotion. Brain Res. 149: 503507.

Andersson, O., H. Forssberg, S. Grillner, and P. Wallen (1981) Peripheral feedback mechanisms acting on the central pattern generators for locomotion in fish and cat. Can. J. Physiol. Pharmacol. 59: 713726.

Ayers, J., and W. J. Davis (1977) Neural control of locomotion in the lobster, Homarus americanus. I. Motor programs for forward and backward walking. J. Comp. Physiol. A 115: 1-27.

Ayers, J., and A. I. Sclverston (1979) Monosynaptic entrainment of an endogenous pacemaker network: A cellular mechanism for von Holst's magnet effect. J. Comp. Physiol. 129: 5-17.

Bacon, J., and M. Tyrer (1979) Wind interneurone input to flight motor neurones in the locust, Schistocerca gregoria. Naturwissenschaften 66: $116-117$

Brodin, L., S. Grillner, and C. Rovainen (1984) NMDA, kainate and quisqualate receptors and the generation of fictive locomotion in the lamprey spinal cord. Brain Res. 325: 302-306.

Cohen, A. H., and P. Wallen (1980) The neuronal correlate of locomotion in fish. "Fictive swimming" induced in an in vitro preparation of the lamprey spinal cord. Exp. Brain Res. 41: 11-18.

Delcomyn, F. (1980) Neural basis of rhythmic behavior in animals. Science 210: 492-498.

Duysens, J. (1977) Reflex control of locomotion as revealed by stimulation of cutaneous afferents in spontaneously walking premammillary cats. J. Neurophysiol. 40: 737-751.

Eisen, J. S., and E. Marder (1984) A mechanism for production of phase shifts in a pattern generator. J. Neurophysiol. 5l: 1375-1393.

Fitch, G. K., and A. E. Kammer (1982) Modulation of the ventilatory rhythm of the hellgrammite Corydalus cornutus by mechanosensory input. J. Comp. Physiol. 149: 423-434.

Grillner, S. (1974) On the generation of locomotion in the spinal dogfish. Exp. Brain Res. 20: 459-470.

Grillner, S. (1985) Neurobiological bases of rhythmic motor acts in vertebrates. Science 228: 143-149.

Grillner, S., and P. Wallen (1982) On peripheral control mechanisms acting on the central pattern generators for swimming in the dogfish. J. Exp. Biol. 98: 1-22.

Grillner, S., and P. Wallen (1984) How does the lamprey central nervous system make the lamprey swim? J. Exp. Biol. 112: 337-357.

Grillner, S., and P. Wallen (1985) The effect of current passage on $N$ - methyl-D-aspartate-induced, tetrodotoxin-resistant membrane potential oscillations in lamprey neurons active during locomotion. Neurosci. Lett. 56: 87-93.

Grillner, S., A. McClellan, and C. Perret (1981a) Entrainment of the spinal pattern generators for swimming by mechano-sensitive elements in the lamprey spinal cord in vitro. Brain Res. 217: 380-386.

Grillner, S., A. McClellan, K. Sigvardt, P. Wallen, and M. Wilen (1981b) Activation of NMDA-receptors elicits "fictive locomotion" in lamprey spinal cord in vitro. Acta Physiol. Scand. 113: 549-551.

Grillner, S., A. McClellan, and K. Sigvardt (1982) Mechanosensitive neurons in the spinal cord of the lamprey. Brain Res. 235: 169-173.

Grillner, S., T. Williams, and P.-A. Lagerbäck (1984) The edge cell, a possible intraspinal mechanoreceptor. Science 223: 500-503.

Grobstein, P. (1973) Extension-sensitivity in the crayfish abdomen I. Neurons monitoring nerve cord length. J. Comp. Physiol. 86: 331348.

Ikeda, K., and C. A. G. Wiersma (1964) Autogenic rhythmicity in the abdominal ganglia of the crayfish: The control of swimmeret movements. Comp. Biochem. Physiol. 12: 107-115.

Martin, A. R., and W. O. Wickelgren (1971) Sensory cells in the spinal cord of the sea lamprey. J. Physiol. (Lond.) 212: 65-83.

McClellan, A. D. (1984) Descending control and sensory gating of "fictive" swimming and turning responses elicited in an in vitro preparation of the lamprey brainstem/spinal cord. Brain Res. 302: 151162.

McClellan, A. D. (1986) Command systems for initiating locomotor responses in fish and amphibians-Parallels to initiation of locomotion in mammals. In Neurobiology of Vertebrate Locomotion, Wenner-Gren Symposium Series, S. Grillner, P. Stein, D. Stuart, H. Forssberg, and R. Herman, eds., pp. 3-20, MacMillan, London. 
McClellan, A. D., and S. Grillner (1983) Initiation and sensory gating of "fictive" swimming and withdrawal responses in an in vitro preparation of the lamprey spinal cord. Brain Kes. 269: 237-250.

McClellan, A. D., and K. A. Sigvardt (1985) Features of entrainment of spinal pattern generators for locomotor activity in the lamprey. Soc. Neurosci. Abstr. 11: 881.

Möhl, B., and W. Nachtigall (1978) Proprioceptive input on the locust flight motor revealed by muscle stimulation. J. Comp. Physiol. 128: $57-65$.

Pearson, K. G., D. N. Reye, and R. M. Robertson (1983) Phasedependent influences of wing stretch receptors on flight rhythm in the locust. J. Neurophysiol. 49: 1168-1181.

Perkel, D. H., J. H. Schulman, T. H. Bullock, G. P. Moore, and J. P. Segundo (1964) Pacemaker neurons: Effects of regularly spaced synaptic inputs. Science 145: 61-63.

Peterson, E. L., and R. L Calabrese (1982) Dynamic analysis of a rhythmic neural circuit in the leech Hirudo medicinalis. J. Neurophysiol. 47: 256-271.

Pinsker, H. M. (1977a) Aplysia bursting neurons as endogenous oscillators. I. Phase-response curves for pulsed inhibitory synaptic input. J. Neurophysiol. 40: 527-543.

Pinsker, H. M. (1977b) Aplysia bursting neurons as endogenous oscillators. II. Synchronization and entrainment by pulsed inhibitory input. J. Neurophysiol. 40: 544-555.

Pinsker, H. M., and J. Ayers (1983) Neuronal oscillators. In Clinical Neurosciences, R. N. Rosenberg, ed., vol. 5: Neurobiology, W. D. Willis, ed., pp. 203-266, Churchill Livingstone, New York.

Poon, M. (1980) Induction of swimming in lamprey by L-DOPA and amino acids. J. Comp. Physiol. 136: 337-344.

Robertson, R. M., and M. Moulins (1981) Control of rhythmic behaviour by a hierarchy of linked oscillators in crustacea. Neurosci. Lett. 21: 111-116.

Robertson, R. M., and K. G. Pearson (1983) Interneurons in the flight system of the locust: Distribution, connections, and resetting properties. J. Comp. Neurol. 215: 33-50.

Rovainen, C. M. (1974) Synaptic interactions of identified nerve cells in the spinal cord of the sea lamprey. J. Comp. Neurol. 154: 189206.
Rovainen, C. M. (1985) Effects of groups of propriospinal interneurons on fictive swimming in the isolated spinal cord of the lamprey. $J$. Neurophysiol. 54: 299-317.

Schroeder, D. M., and S. C. Richardson (1985) Is the intimate relationship between ligaments and marginal specialized cells in the snake's spinal cord indicative of a CNS mechanoreceptor? Brain Res. 328 . 145-149.

Selzer, M. E. (1979) Variability in maps of identified neurons in the sea lamprey spinal cord examined by a wholemount technique. Brain Res. 163: 181-193.

Sigvardt, K. A., S. Grillner, P. Wallen, and P. A. M. van Dongen (1985) Activation of NMDA receptors elicits fictive locomotion and bistable membrane properties in the lamprey spinal cord. Brain Res. 336 390-395.

Stein, P. S. G. (1971) Intersegmental coordination of swimmeret motoneuron activity in crayfish. J. Neurophysiol. $34: 310-318$.

Stein, P. S. G. (1976) Mechanisms of interlimb phase control. In Neural Control of Locomotion, R. M. Herman, S. Grillner, P. Stein, and D. Stuart, eds., pp. 465-487, Plenum, New York.

Tang, D., and M. E. Selzer (1979) Projections of lamprey spinal neurons determined by the retrograde axonal transport of horseradish peroxidase. J. Comp. Neurol. 188: 629-646.

Wallen, P., and T. L. Williams (1984) Fictive locomotion in the lamprey spinal cord in vitro compared with swimming in the intact and spinal lamprey. J. Physiol. 347: 225-239.

Wallen, P., and T. L. Williams (1985) The role of movement-related feedback in the control of locomotion in fish and lamprey. In Feedback and Motor Control in Invertebrates and Vertebrates, W. J. P. Barnes and M. H. Gladden, eds., pp. 317-335, Croom Helm, London.

Wendler, G. (1974) The influence of proprioceptive feedback on locust flight co-ordination. J. Comp. Physiol. 88: 173-200.

Wickelgren, W.O. (1978) Physiological and anatomical characteristics of reticulospinal neurons in lamprey. J. Physiol. (Lond.) 270: 89-114.

Young, R. E., and P. E. Coyer (1979) Phase coordination in the cardiac and ventilatory rhythms of the lobster Homarus americanus. J. Exp. Biol. 82: 53-74. 